\title{
Evaluation of Inflammatory Markers in Pregnant Women at Risk, for the Prediction of Preeclampsia
}

\author{
Oancea Mihaela Daniela, Costin N, Pop Daria Maria, Ciortea R, Trif loana, Mihu D \\ Ind Obstetrics and Gynecology Department, luliu Hațieganu University of Medicine and Pharmacy, Cluj-Napoca, Romania
}

\begin{abstract}
Introduction: A low degree of inflammation has been associated with complications in pregnancies, including preeclampsia (PE). The aim of our study was to determine the serum values of high sensitivity C Reactive Protein (hs-CRP) and Interleukin-6 (IL-6) in the first and second trimesters of pregnancy in pregnant women with risk factors for the development of PE, and to evaluate their relevance for the prediction of this disorder.

Material and methods: We performed a prospective longitudinal study on 120 pregnant women, who were divided based on the pregnancy evolution, into two groups: group I - 26 pregnant women who developed preeclampsia and group II - 94 pregnant women with physiological evolution of pregnancy.

Results: Our study has shown an increase in serum levels of hs-CRP and IL-6 in the first and second trimester of pregnancy in patients from group I, significant values being revealed only in the second trimester of pregnancy. The predictive power of the selected inflammatory markers was significant only for values of hs-CRP in the second trimester of pregnancy, while the association with IL-6 increased the prediction. Conclusions: Increased values of hs-CRP and IL-6 in the second trimester of pregnancy are associated with higher risk for preeclampsia, however the study provided only a modest efficiency of the prediction capacity.
\end{abstract}

Keywords: prediction, preeclampsia, IL-6, hs-CRP

Received: 7 February 2014 / Accepted: 8 May 2014

\section{Introduction}

Preeclampsia (PE) is a multisystemic disorder specific to human pregnancy, which remains an important cause of materno-fetal morbidity and mortality, requiring considerable efforts to find effective prevention strategies. Although the etiopathogeny of this syndrome remains unclear, it is certain that PE is triggered by placental factors given that it occurs only in pregnant women, and definitive treatment is delivery [1].

There is an immunological theory that supports trophoblast and spiral artery remodeling alteration by immune cells, determining the release of ischemic placental factors in the systemic maternal circulation. Also, the increase in the levels of angiogenic factors and trophoblastic detritus may play a role in the pathogeny of PE. In addition to these hypotheses, the imbalance between pro- and antiinflammatory factors has also been incriminated in the etiopathogeny of this disorder [2].

The factors that generate an inflammatory response in $\mathrm{PE}$ are represented by the activation of circulating leukocytes, the increase of the production of neutrophil activators and complement activity. The exposure of endothelial cells to proinflammatory cytokines induces an activation of the coagulation system, of platelet aggregation, vasospasm and endothelial activation [3,4].

Recent studies have evidenced a small degree of inflammation in normal pregnancy. It is not yet known to

Correspondence to: Daria Maria Pop

E-mail: dariagroza@yahoo.com what extent excessive maternal inflammation causes endothelial dysfunction in patients that subsequently develop PE [5]. The most extensively studied inflammatory markers include $\mathrm{C}$ reactive protein (CRP), interleukin 6 (IL-6), interleukin 8 (IL-8), interleukin 10 (IL-10) and tumor necrosis factor alpha (TNF- $\alpha$ ). The available data suggest the role of these in the mechanisms responsible for the development of PE, but are ambiguous regarding their predictive value $[4,6,7]$. CRP is an acute phase reagent and the most sensitive marker of generalized inflammatory activity in the organism $[8,9]$. IL-6 is a multifunctional proinflammatory cytokine, produced by vascular endothelial cells, the placenta and leukocytes, with a decisive role in inflammatory response and the control of T cell differentiation in adaptive immunity. IL- 6 is also involved in trophoblast proli-feration and invasion and in oxidative stress, being the key circulating marker of endothelial dysfunction [10-12].

The aim of our study was to determine the serum values of high sensitivity CRP (hs-CRP) and IL-6 in the first and second trimesters of pregnancy in pregnant women with risk factors for the development of PE, and to evaluate their relevance for the prediction of this disorder.

\section{Material and method}

We performed a prospective longitudinal study on 120 pregnant women with risk factors for preeclampsia, who presented to the "Dominic Stanca" Clinic of Obstetrics and Gynecology, Cluj-Napoca, Romania, in the period January 2011 - July 2013. 
The criteria of inclusion in the study were the presence of at least one of the following risk factors: primiparity; a history of preeclampsia in previous pregnancies; a family history of preeclampsia; pregnancy-associated disorders (renal disease, diabetes mellitus, etc.); obstetric history (preeclampsia, fetal hypotrophy, oligoamnios, perinatal mortality, premature detachment of normally inserted placenta); obesity; young age or age over 40 years; low socioeconomic standard.

The exclusion criteria were: pregnant women with clinically manifest infections; recent treatment with non-steroidal antiinflammatory drugs and corticosteroids (in the last 14 days); chronic autoimmune and inflammatory diseases.

All patients included in the study signed an informed consent and the study was approved by the Ethics Board of the "Iuliu Hațieganu" University of Medicine and Pharmacy of Cluj-Napoca. The study investigated the serum values of hs-CRP and IL-6 in the first trimester of pregnancy (weeks 10-14) and in the second trimester of pregnancy (weeks 20-24). The samples were analyzed according to the following study protocol: $3 \mathrm{ml}$ blood were collected on anticoagulant, the plasma was separated by centrifugation for $15 \mathrm{~min}$ at $1000 \mathrm{xg}$. The samples were stored in the freezer at $-20^{\circ} \mathrm{C}$. When processed, they were gradually thawed on ice. The samples were processed according to the instructions of the CRP HS ELISA (DRG Instruments GmbH, Germany) and Human IL-6 Quantikine ELISA kits (R\&D Systems Europe Ltd., Abingdon OX143NB, $\mathrm{UK})$.

The pregnant women were monitored periodically until delivery, and the development of PE or the physiological evolution of pregnancy was assessed. For the diagnosis of PE, we used the classification system proposed by U.S. NMBPEP Working Group 2000, preceded by AGOC: blood pressure values of at least $140 / 90 \mathrm{mmHg}$ (on 2 examinations minimum $6 \mathrm{~h}$ and maximum 7 days apart), proteinuria higher than $30 \mathrm{mg} / \mathrm{dl}$ (in 2 urine samples collected 4-6 h apart); high blood pressure and proteinuria occurred after 20 weeks of pregnancy in a pregnant woman that was normotensive prior to pregnancy and were normalized by 12 weeks postpartum.

At the end of the study, the patients were divided into two groups depending on the evolution of pregnancy:

Group I - 26 pregnant women who developed preeclampsia: 9 the severe form, and 17 the moderate form. Of the 9 pregnant women with severe preeclampsia, 4 also had intrauterine growth restriction (IUGR).

Group II - 94 pregnant women who did not develop preeclampsia and had a physiological evolution of pregnancy.

\section{Statistical analysis}

We used the Mann-Whitney test for the comparison of IL- 6 and hs-CRP values in the group with preeclampsia compared to the control group. The $\mathrm{t}$-test was inadequate in this case, because the IL- 6 and hs-CRP values did not have a normal distribution. We used the binary logit model to assess whether IL- 6 and hs-CRP values were correlated with the probability of developing preeclampsia. We noted $Y=1$ if the patient had preeclampsia and $\mathrm{Y}=0$ if the patient did not have preeclampsia. The t-test was used for the study of the statistical significance of the coefficients. We considered that a variable was statistically significant if the associated $\mathrm{p}$ value was $<0.05$. The predictive power of the functions used was evaluated using the ROC (Receiver Operating Characteristics) curve. All statistical processings were performed with the STATA 9.1 software (StataCorp, 4905 Lakeway Drive, College Station, Texas 77845 USA).

\section{Results}

We observed that patients with preeclampsia have higher mean and median IL- 6 and hs-CRP values. The differences are more significant for hs-CRP. In the second trimester, both IL-6 and hs-CRP values are more significantly increased than in the first trimester.

\section{Predictive significance of IL-6 values in the first trimes- ter}

The slightly increased IL- 6 values in the first trimester are not statistically significant. The sign of the coefficient is as expected, positive, and is also found in similar studies.

The predictive power of IL- 6 values in the first trimester is low $($ AUROC $=0.6277)$. Combined with the regression result, it shows that a suspicion of preeclampsia based only on IL-6 values in the first trimester is poorly supported.

\section{Predictive significance of IL-6 values in the second tri- mester}

The IL- 6 value in the second trimester is statistically significant at a 5\% threshold. The coefficient has the expected sign, positive. Consequently, a higher IL- 6 value in the $2^{\text {nd }}$ trimester increases the probability of preeclampsia.

The predictive power of IL- 6 values in the second trimester is only slightly higher $($ AUROC $=0.6978)$ than that of IL- 6 values in the first trimester. However, it remains rather low. A diagnosis of preeclampsia cannot be made only based on IL- 6 values in the second trimester,

Table I. Comparative IL-6 and hs-CRP values ( $p$-value according to the Mann-Whitney test)

\begin{tabular}{lccccc}
\hline Variable & Group & Mean & Median & $\begin{array}{c}\text { Standard } \\
\text { deviation }\end{array}$ & p-value \\
\hline IL-6-1 st trimester $^{\text {Group I }}$ & 4.63 & 4.65 & 0.22 & 0.121 \\
& Group II & 4.54 & 4.49 & 0.27 & \\
IL-6-2 nd trimester $^{\text {Group I }}$ & 4.66 & 4.68 & 0.34 & 0.096 \\
hs-CRP - 1st & Group II & 4.50 & 4.47 & 0.22 & \\
trimester & Group I & 0.56 & 0.49 & 0.36 & 0.015 \\
hs-CRP -2 2nd & Group II & 0.41 & 0.26 & 0.57 & \\
trimester & Group I & 0.91 & 0.70 & 0.54 & 0.001 \\
& Group II & 0.51 & 0.40 & 0.61 & \\
\hline
\end{tabular}


Table II. Results of binary logit regression, with the explanatory variable IL-6 in the $1^{\text {st }}$ trimester

\begin{tabular}{lcccc}
\hline & Coefficient & $\begin{array}{c}\text { Standard } \\
\text { error }\end{array}$ & $z$ & $p$-value \\
\hline IL-6 $-1^{\text {st }}$ trimester & 1.22029 & 1.01508 & 1.20 & 0.229 \\
Constant & -7.61233 & 4.69082 & -1.62 & 0.105 \\
\hline
\end{tabular}

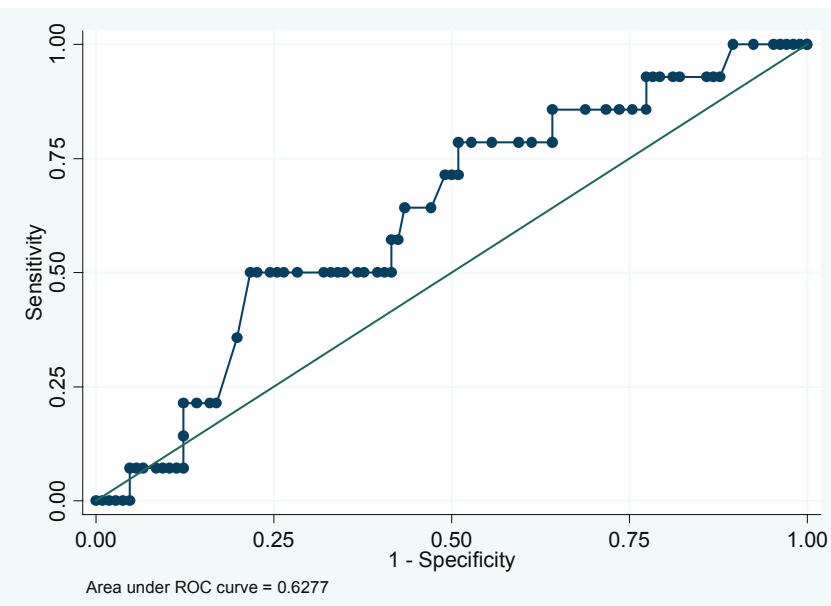

Fig. 1. The ROC curve associated with the binary logit model. Explanatory variable: IL-6 in the $1^{\text {st }}$ trimester

Table IV. Results of binary logit regression, with the explanatory variable hs-CRP in the $1^{\text {st }}$ trimester

\begin{tabular}{lcccc}
\hline & Coefficient & $\begin{array}{c}\text { Standard } \\
\text { error }\end{array}$ & $z$ & p-value \\
\hline hs-CRP - 1st trimester & 0.36886 & 0.40983 & 0.90 & 0.368 \\
Constant & -2.19969 & 0.35732 & -6.16 & 0.000 \\
\hline
\end{tabular}

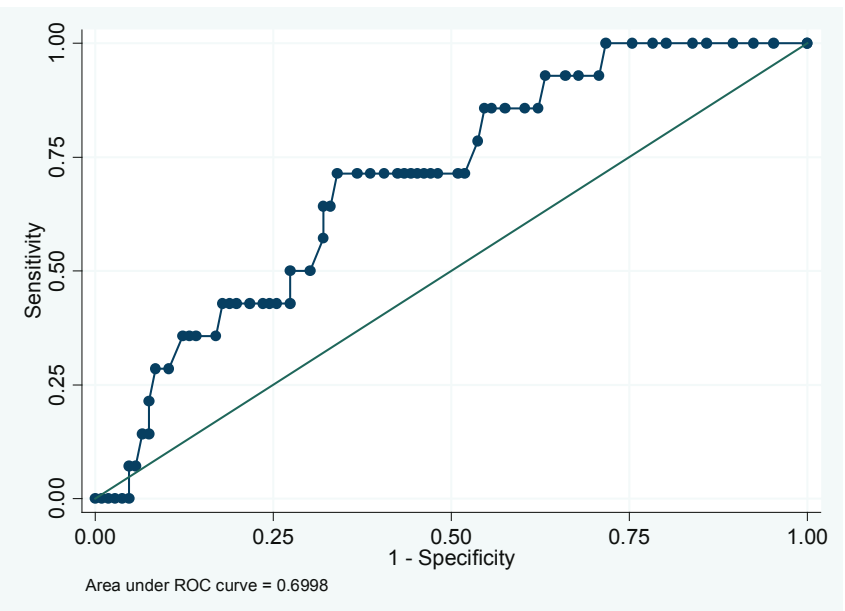

Fig. 3. The ROC curve associated with the binary logit model. Explanatory variable: hs-CRP in the $1^{\text {st }}$ trimester

predictability being modest. It is recommended to take into consideration other factors to support a serious suspicion of preeclampsia.

\section{Predictive significance of hs-CRP values in the first tri-} mester

The hs-CRP value in the first trimester is not statistically
Table III. Results of binary logit regression, with the explanatory variable IL-6 in the $2^{\text {nd }}$ trimester

\begin{tabular}{lcccc}
\hline & Coefficient & $\begin{array}{c}\text { Standard } \\
\text { error }\end{array}$ & $z$ & $p$-value \\
\hline IL-6 $-2^{\text {nd }}$ trimester & 2.63622 & 1.16294 & 2.27 & 0.023 \\
Constant & -17.0949 & 5.39779 & -2.61 & 0.009 \\
\hline
\end{tabular}

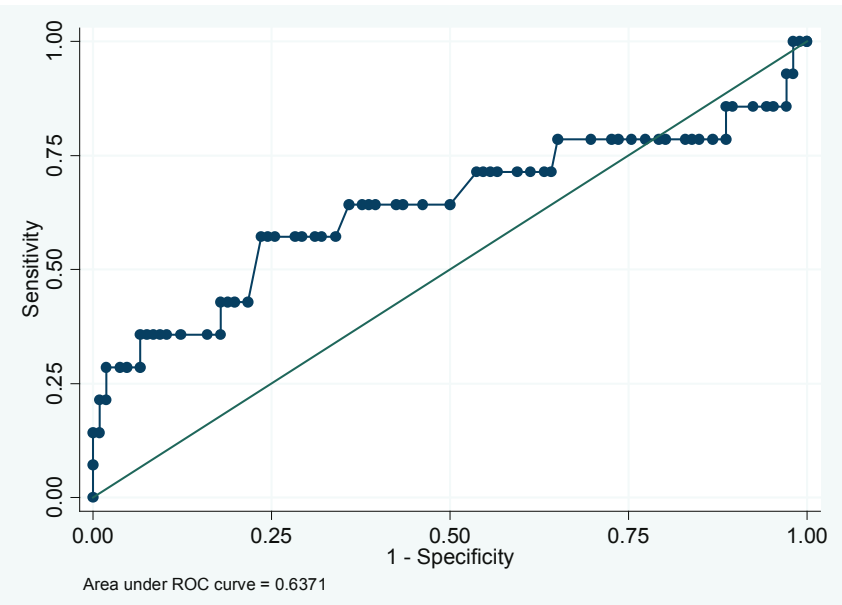

Fig. 2. The ROC curve associated with the binary logit model. Explanatory variable: IL- 6 in the $2^{\text {nd }}$ trimester

Table V. Results of binary logit regression, with the explanatory variable $\mathrm{hs}-\mathrm{CRP}$ in the $2^{\text {nd }}$ trimester

\begin{tabular}{lcccc}
\hline & Coefficient & $\begin{array}{c}\text { Standard } \\
\text { error }\end{array}$ & $z$ & $p$-value \\
\hline hs-CRP - 2nd trimester & 0.71567 & 0.35338 & 2.03 & 0.043 \\
Constant & -2.19969 & 0.35732 & -6.16 & 0.000 \\
\hline
\end{tabular}

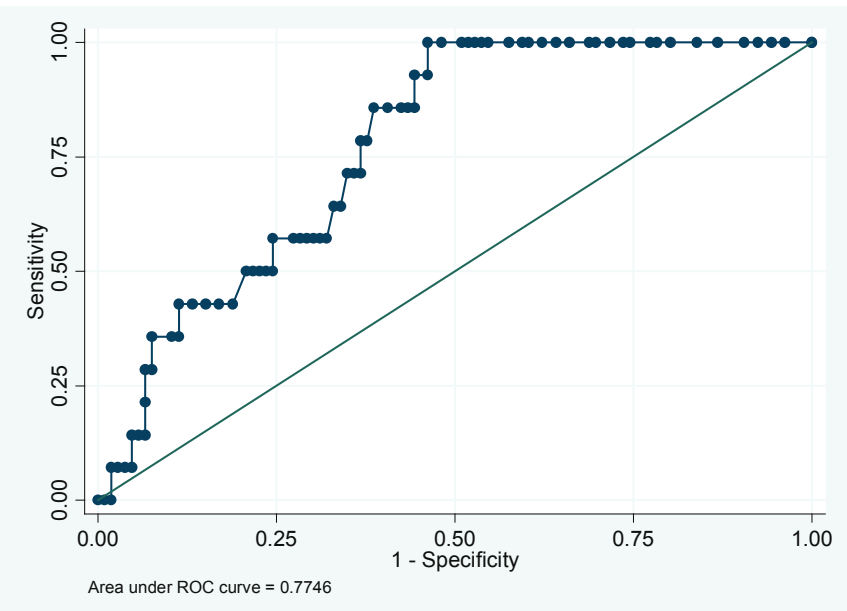

Fig. 4. The ROC curve associated with the binary logit model. Explanatory variable: hs-CRP in the $2^{\text {nd }}$ trimester

significant. The coefficient has a positive sign, according to previous empirical studies, but because it is insignificant, no conclusion regarding the influence of hs-CRP in the first trimester on the probability of preeclampsia can be drawn.

The predictive power of hs-CRP values in the first trimester is moderate (AUROC $=0.6998$ ). Higher hs-CRP 
Table VI. Results of binary logit regression, with the explanatory variables IL- 6 and hs-CRP in the $1^{\text {st }}$ trimester

\begin{tabular}{lcccc}
\hline & Coefficient & $\begin{array}{c}\text { Standard } \\
\text { error }\end{array}$ & $z$ & p-value \\
\hline IL-6 $-1^{\text {st }}$ trimester & 1.04218 & 1.06040 & 0.98 & 0.326 \\
hs-CRP $-1^{\text {st }}$ trimester & 0.23487 & 0.43828 & 0.54 & 0.592 \\
Constant & -6.90641 & 4.82629 & -1.43 & 0.152 \\
\hline
\end{tabular}

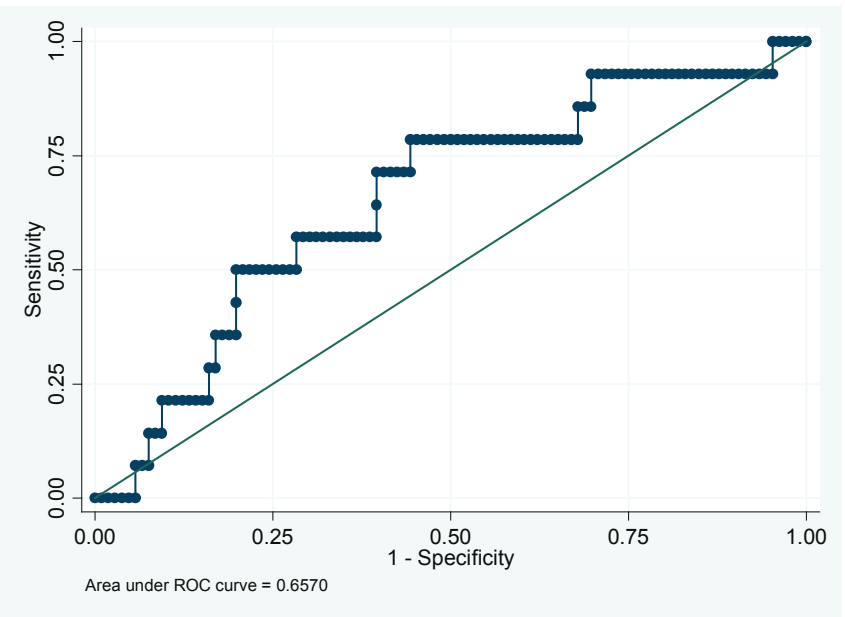

Fig. 5. The ROC curve associated with the binary logit model. Explanatory variable: IL-6 in the $1^{\text {st }}$ trimester

values in the first trimester indicate the probability of preeclampsia.

\section{Predictive significance of hs-CRP values in the second trimester}

The hs-CRP value in the second trimester is statistically significant at a 5\% threshold. Increased hs-CRP values in the second trimester indicate a clear risk for the development of preeclampsia.

The predictive power of hs-CRP values in the second trimester is high (AUROC $=0.7746$ ). Higher hs-CRP values in the second trimester are a serious indicator of the probability of preeclampsia. The percentage of correct predictions based on hs-CRP values in the second trimester is about $77 \%$.

\section{Predictive significance of IL-6 and hs-CRP values in the first trimester}

In multiple regression analysis, the coefficients for IL-6 and hs-CRP in the first trimester also remain statistically insignificant. A prediction of preeclampsia based on these results would be poorly supported.

The predictability of preeclampsia based on IL- 6 and hs-CRP in the first trimester is not very accurate, which confirms the conclusions derived from regression.

\section{Predictive significance of IL-6 and hs-CRP values in the second trimester}

In multiple regression analysis, the coefficients for IL- 6 and hs-CRP in the second trimester also maintain their statistical significance at a 5\% and 10\% threshold, respectively.
Table VII. Results of binary logit regression, with the explanatory variables IL- 6 and hs-CRP in the $2^{\text {nd }}$ trimester

\begin{tabular}{lcccc}
\hline & Coefficient & $\begin{array}{c}\text { Standard } \\
\text { error }\end{array}$ & $z$ & $p$-value \\
\hline IL-6 - 1st trimester & 2.45818 & 1.16144 & 2.12 & 0.034 \\
hs-CRP $-1^{\text {st }}$ trimester & 0.66744 & 0.35710 & 1.87 & 0.062 \\
Constant & -13.7244 & 5.38652 & -2.55 & 0.011 \\
\hline
\end{tabular}

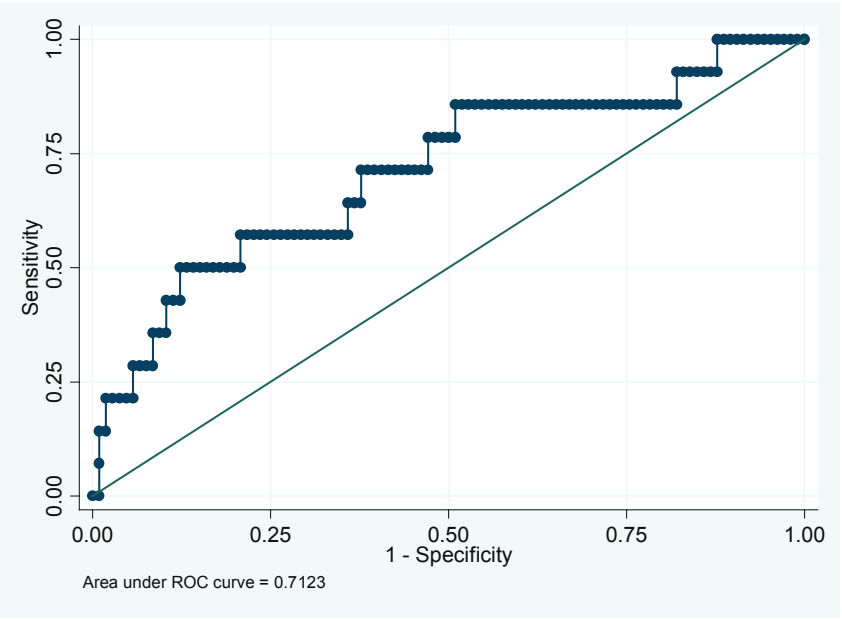

Fig. 6. The ROC curve associated with the binary logit model. Explanatory variable: IL- 6 in the $1^{\text {st }}$ trimester

This is a good premise to try a prediction for the development of preeclampsia based on their conjugated values.

The predictability of preeclampsia based on IL- 6 and hsCRP in the second trimester is superior to the one based on the same indicators in the first trimester.

\section{Discussion}

The immune system and inflammation play an important role in implantation, placentation and labor, and it is known that the pathogenesis of PE is at least in part immunologically mediated. PE is characterized by a proinflammatory status at the level of the fetomaternal interface, which determines an orientation towards Th 1 immune response, compared to antiinflammatory response in normal pregnancy [5]. The clinical interest in inflammatory markers in pregnancy led to the investigation of the acute phase protein hs-CRP and IL- 6 in the first and second trimesters of pregnancy in pregnant women with risk factors for the development of PE.

Our results indicate an increase of serum IL-6 levels in PE compared to normal pregnancy, with more significantly increased values in the second trimester. Similar data were reported by other literature studies which suggest a marked inflammatory response in PE [13-16].

The plasma activation of endothelial cells in PE stimulates IL-6 production that is inhibited by vitamin E [17]. Other recent studies suggest that IL-6 levels in the placenta and peripheral blood do not play a significant role in the induction of an immunological imbalance that leads to PE $[13,18]$. The association of high IL-6 levels with the development of PE has a moderate predictive power only 
in the second trimester of pregnancy, in the first trimester predictability being reduced.

Currently, it is not known whether IL-6 plays a direct role in the pathogeny of PE or is just a marker of endothelial cell activation. Recently, it has been demonstrated that IL-6 can interfere with the mechanisms of contraction and relaxation at the level of systemic vessels in pregnancy, playing a role in modeling vascular resistance and implicitly blood pressure [19].

Our study detected increased hs-CRP values in the first and second trimesters of pregnancy in pregnant women who subsequently developed $\mathrm{PE}$, a significant increase of hs-CRP being evidenced in the second trimester. The data obtained by Wolf et al. and Tjoa et al., who found increased CRP levels as early as the first trimester in pregnancies that subsequently developed PE, are in accordance with our results $[20,21]$. There are also contradictory data resulting from the studies of Savvidou, who found no differences between CRP levels in pregnant women with PE compared to normal pregnancy $[4,22,23]$.

The results of our study indicate the association of high hs-CRP levels in the first and second trimesters of pregnancy with the development of $\mathrm{PE}$, the serum hs-CRP values obtained in the second trimester having a higher predictive power. For a better and early prediction of PE, we associated the two inflammatory markers studied in the first and second trimesters of pregnancy, the predictive power of the conjugated hs-CRP and IL- 6 values being higher in the second trimester.

\section{Conclusions}

The obtained data suggest the presence of a generalized inflammatory response in patients with $\mathrm{PE}$ as early as the first trimester of pregnancy.

The evaluation of inflammatory markers in pregnancy has complex implications regarding the prediction, screening and prophylaxis of PE. The increased hs-CRP and IL-6 values in the second trimester of pregnancy are associated with a higher risk to develop PE, the study providing only a modest efficiency of the prediction capacity. Further investigations of additional factors that might improve the prediction rate are justified in order to develop more accurate screening methods.

\section{References}

1. Catarino C, Santos-Silva A, Belo L, et al. Inflammatory Disturbances in Preeclampsia: Relationship between Maternal and Umbilical Cord Blood. J Pregnancy. 2012;2012: Article ID 684384.

2. Xiao JP, Yin YX, Gao YF, et al. The increased maternal serum levels of $\mathrm{IL}-6$ are associated with the severity and onset of preeclampsia. Cytokine 2012;60:856-860.
3. Ouyang Y, Li SJ, Zhang Q, Cai H, Chen HP. Interaction Between Inflammatory and Oxidative Stress in Preeclampsia. Hypertens Pregnancy. 2009;28:56-62.

4. Guven M, Coskun A, Ertas IE, et al. Association of Maternal Serum CRP, IL-6, TNF- $\alpha$, Homocysteine, Folic Acid and Vitamin B12 Levels with the Severity of Preeclampsia and Fetal Birth Weight. Hypertens Pregnancy. 2009;28:190-200.

5. Haedersdal S, Salvig J, Aabye M, et al. Inflammatory Markers in the Second Trimester Prior to Clinical Onset of Preeclampsia, Intrauterine Growth Restriction, and Spontaneous Preterm Birth. Inlammation. 2013;4:907-913.

6. Babu MS, Bobby Z, Habeebullah S. Increased inflammatory response and imbalance in blood and urinary oxidant-antioxidant status in South Indian women with gestational hypertension and preeclampsia. Clin Biochem. 2012;45:835-838.

7. Mihu D, Sabau L, Costin N, Ciortea R, Malutan A, Mihu MC. Implication of maternal systemic oxidative stress in normal pregnancy and in pregnancy complicated by preeclampsia. Journal of maternal - Fetal and Neonatal Medicine. 2012;27(7):944-951.

8. Kumru S, Godekmerdan A, Kutlu S, Ozcan Z. Correlation of maternal serum high-sensitive C-reactive protein levels with biochemical and clinical parameters in preeclampsia. Eur $\mathrm{J}$ Obstet Gynecol Reprod Biol. 2006;124:164-167.

9. Mihu D, Costin N, Sabau L, et al. Implications of serum C-Reactive Protein in normal pregnancy and preeclampsia. Obstetrica si ginecologia. 2011; LIX(Supl): 60-67.

10. Prins J, Gomez-Lopez N, Robertson SA. Interleukin-6 in pregnancy and gestational disorders. J Reprod Immunol. 2012;95:1-14.

11. Ozler A, Turgut A, Sak ME, et al. Serum levels of tumor necrosis factor alpha and Interleukin-6 in preeclampsia: relationship with disease severity. Eur Rev Med Pharmacol Sci. 2012;16:1707-1712.

12. LeMarca B, Brewer J, Wallace K. IL-6-induced pathophysiology during pre-eclampsia: potential therapeutic role for magnesium sulfate? Int J Infereron Cytokine Mediator Res. 2011;3:59-64.

13. Stonek F, Hafner E, Metzenbauer M, et al. Absence of an association of tumor necrosis factor (TNF)-alpha and interleukin-10 (IL-10) G1082A polymorphism in women with preeclampsia. J Reprod Immunol. 2008;77:85-90.

14. Jonsson $Y$, Ruber M, Matthiesen L, et al. Cytokine mapping of sera from women with preeclampsia and normal pregnancies. J Reprod Immunol. 2006;70:83-91.

15. Luppi $P$, Tse $H$, Lain $K Y$, et al. Preeclampsia activates circulating immune cells with engagement of the NF-kappaB pathway. Am J Reprod Immunol. 2006;56:135-144.

16. Freeman DJ, McManus F, Brown F, et al. Short and long-term changes in plasma inflammatory markers associated with preeclampsia. Hypertension. 2004;44:708-714.

17. Tukacs P, Green KL, Nikaeo A, Kauma SW. Increased vascular endothelial cell production of interleukin-6 in severe preeclampsia. Am J Obstet Gynecol. 2003;188:740-744.

18. Hayashi M, Ueda Y, Ohkura T, Inaba N. Interleukin-6 concentration in the placenta and blood in normal pregnancies and preeclampsia. Horm. Metab. Res. 2005;37:419-424.

19. Mihu D, Costin N, Sabau L, et al. Interleukina-6 - marker al sindromului inflamator in preeclampsie. Gineco.ro. 2010;22:230-234.

20. Wolf M, Kettyle L, Sandler L, et al. Obesity and preeclampsia: The potential role of inflammation. Obstet Gynecol. 2001;98:757-762.

21. Tjoa ML, van Vugt JM, Go AT, Blankenstein CB, Oudejans CB. Elevated $\mathrm{C}$-reactive protein levels during first trimester of pregnancy are indicative of preeclampsia and intrauterine growth restriction. J Reprod Immunol. 2003;59:29-37.

22. Savvidou MD, Lees CC, Parra M, Hingorani AD, Nicolaides KH. Levels of C-reactive protein in pregnant women who subsequently develop preeclampsia. BJOG. 2002;109:297-301.

23. Djurovic S, Clausen T, Wergeland R, et al. Absence of systemic inflammatory response at 18 weeks of gestation in women with subsequent preeclampsia. BJOG. 2002;109:759-764. 